\title{
Potential Role of Knee Print in Forensic Identification
}

\section{Nagy M. B. Al-Fadaly*}

Department of Forensic Medicine and Clinical Toxicology, Faculty of Medicine, Al-Azhar University, Cairo, Egypt

\begin{abstract}
In this paper, an initial method of collecting knee print and explaining how to prepare it, has been presented. The results of this pilot experiment, which was carried out in order to study the possibility of identifying persons from their knee prints, were also presented. The results showed that by adopting this method, knee print has shown a considerable degree of variability among different individuals. The variability of knee print among different individuals and its potential role in personal identification for forensic field work, particularly, in cases when knee print is involved by some way or another has been discussed. Finally the knee print can probably be considered in the forensic field. Furthermore, more research studies on the knee print should be carried out as it might potentially become a useful forensic identification tool.
\end{abstract}

\section{Introduction}

In forensic field, individual identification is still the most challenging problem that forensic investigator can encounter. This is not a problem when different kinds of evidentiary items are available. However, this can be a formidable task when no evidence could be found. Adding the knee print to the forensic arsenal can probably broaden the scope of individual identification. I was wandering about the knee print and really surprised by the absence of any studies (references or sources) published about it and about its possible role in forensic identification.

Among all the biometric techniques, fingerprint-based identification is the oldest method which has successfully been used in numerous applications. Everyone is known to have unique, immutable fingerprints [1]. Fingerprint identification is based on two basic premises: (i) persistence: the basic characteristics of fingerprints do not change with time; and (ii) individuality: the fingerprint is unique to an individual [8].

Fingerprint is made of a series of ridges and grooves on the palmar surface of the fingers. The uniqueness of a fingerprint can be determined by the pattern of ridges and grooves as well as the minute points. The minute points are local ridge characteristics that occur at either a ridge bifurcation or a ridge ending [2,3].

Forensic friction ridge (i.e. fingerprint, palmprint, footprint) examination (FFRE) has long been recognized and accepted as the standard for personal identification worldwide [4].

Other forms of prints are also available in the forensic arsenal such as ear prints, iris prints and lip prints, which are used as forensic tools of identification [5].

It is common that forensic pathologist comes across cases in which such prints are not available or mutilated as in case of burnt or dismembered bodies. Probably it is important to find a certain way to be able to identify each part of the human body, particularly for cases in which the forensic pathologist is asked to identify dismembered body parts.

This paper is presenting a new body print that has proven for some degree to be useful in personal identification, especially in cases in which the knee print is involved while other prints are not available or non-identifiable.

The skin of anterior surface of knee joint (patellar surface) bears flattened ridges and linear grooves in between and both form what can be called knee print. Unfortunately, knee print has never been mentioned by any published source as a potential identification tool (up to my knowledge) and we could not find any source about it.

I has been realized that patellar surface of the knee bears a print that would be variable among different individuals and can probably be used for personal identification especially in cases in which knee is involved by a way or another.

I would like to confirm again that I looked several times for any reference regarding the knee print and tried on most if not all known search engines (such as Medscape, Medline) to find any author who had mentioned any thing in a book or paper connecting knee print to personal identification but all I got was irrelevant and was about the knee joint; diseases affecting it; its operations and also the knee skin and diseases affecting it [6,7].

\section{Materials and Methods}

In the present study, the potential role of the knee print was studied and estimated. 56 knee prints were collected from the patellar surface of right knees of 56 adult male volunteers (Main group , 56 prints). From the main group, 20 prints were randomly selected, given code letters and considered as positive control group, 20 prints.

Extra 20 knee prints were collected from another group of adult male volunteers, who were different from the individuals of the main group and even not related to them, given the name (negative control group, 20 prints) and code letters were given to them.

Both of the 20 positive control samples and 20 negative control samples were mingled well, aiming not to be recognized from each others when used for comparison.

*Corresponding author: Nagy M. B. Al-Fadaly, Department of Forensic Medicine and Clinical Toxicology, Faculty of Medicine, Al-Azhar University, Cairo, Egypt, E-mail:nmbayoumy@yahoo.co.uk

Received May 04, 2011; Accepted June 15, 2011; Published June 22, 2011

Citation: Al-Fadaly NMB (2011) Potential Role of Knee Print in Forensic Identification. J Forensic Res 2:131. doi:10.4172/2157-7145.1000131

Copyright: ( 2011 Al-Fadaly NMB. This is an open-access article distributed under the terms of the Creative Commons Attribution License, which permits unrestricted use, distribution, and reproduction in any medium, provided the original author and source are credited. 
All prints of the main and two control groups were magnified and to make the examination easy and simple, each print was divided roughly by 5 transverse and 4 vertical lines into 30 squares or rectangular areas (actually areas vary from 25 - 30 areas), as sometimes some areas are missing.

The rectangular areas of the mixed sample prints of both positive and negative control groups were checked meticulously and compared one by one to that of the 56 sample prints of the main group.

The aim was to identify the identical areas, which could help in identifying comparable prints originated from the same knee.

The knees, during the collection of the prints, were fixed in the fully flexed position. Prints were collected using a bluish violet sealing ink. Prints were collected on A4 paper that is commonly used in Egypt for printing. Each print was collected 4 times and the most clear and visible one was used for comparison. Commonly one or more of the knee prints are partially or completely obliterated during the collection process. Collected knee prints were scanned into the computer and digitally processed using computer software picture programs.

\section{Results}

Naked eye examination of knee prints without any magnification was carried out. Each print per se looked as an irregular sphere of interlacing lines with some irregularities at the periphery representing the projections and indentations, which are common with kneeprints.

Looking at knee prints by the naked eye revealed no or a few differences between such prints. Also the naked eye examination of the rectangular areas dividing the knee print could not reveal the differences that were observed after magnification.

Only, knee print can readily be identified by naked eye examination when it contains a specific mark such as scar of an old wound or operation.

Although many prints showed some areas that were not suitable for identification due to obliteration of its details, they had at least other areas which could be examined and compared, and it was not extremely difficult to (include) match the typical rectangular areas of the same origin print or to exclude the non comparable areas of the different origin prints or even the different (non-corresponding) rectangular areas of the same print. So, identification was carried out after print magnification using computer programs for picture viewing such as paint program (Figure 1)

Prints examination revealed that knee prints commonly include some obliterated or missing areas, which are either as few in number as $1-4$, or as several as $10-15$ areas. This obliteration is most probably caused by any slight movement during collection of the prints. Also missing or obliterated areas can be resulted from existence of projections, caused by bony prominences, or existence of missing areas, caused by depressions, at the periphery or even at the center of the patellar surface of the knee. However, the remaining areas of the print, which were clear, were suitable for examination, identification and comparison.

For comparison, each two areas were displayed on computer desktop side by side and examined meticulously by the naked eye after being magnified. It was a standard rule to keep the known print of the main group always on the left side and the questionable ones of the two control groups on the right side, which were then examined, compared one by one in detail until we got its typical one.
From the sum of 40 sample prints of the two control groups, only 14 could be identified and the rest could not be identified based on typicality. Afterwards, the given code letters were decoded, numbers were checked. It was revealed that the 14 prints identified were from the 20 positive control group and the 26 non-identified sample prints were the remainder 6 prints of the positive control group and the 20 prints of the negative control group.

The single source prints were considered to be matching when one or more of the rectangular areas looked typical. Typical match was finally considered when two rectangular areas proved to be of exact

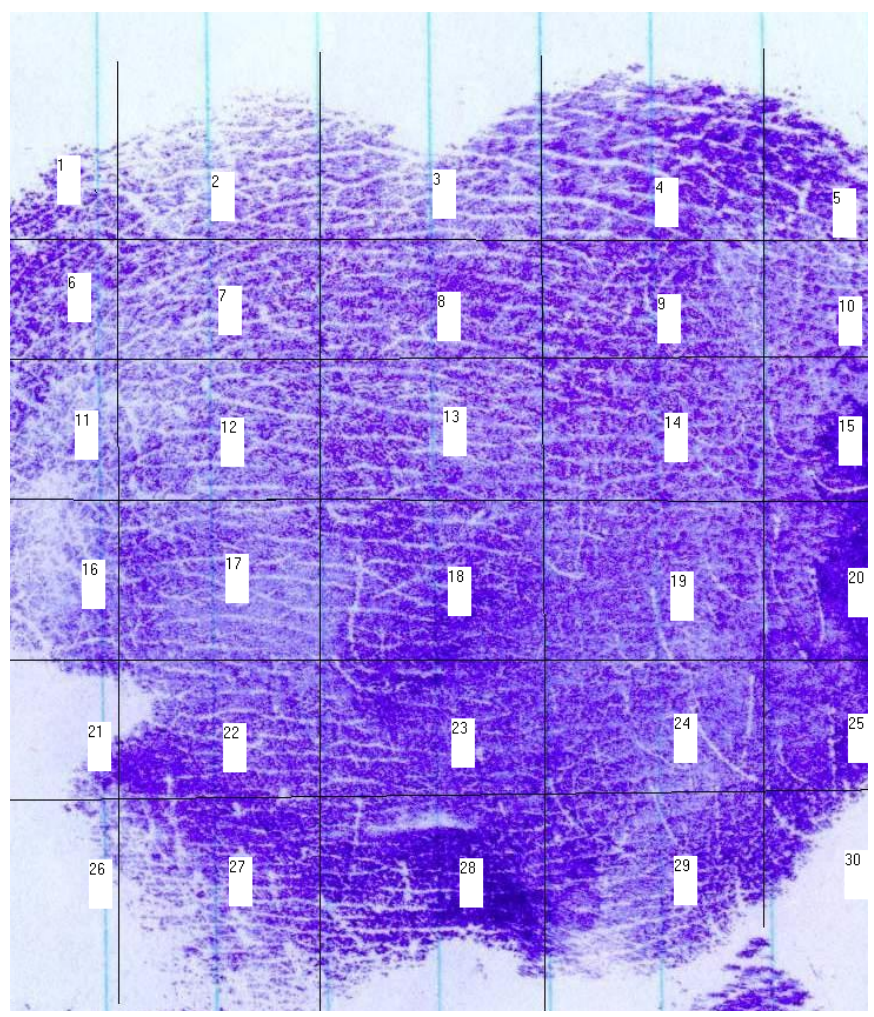

Figure 1: Knee print looks as one unit and to be compared, it can be divided under computer software magnification programes into 30 square or rectangular areas by 4 transverse and 5 vertical lines. The areas are numbered 1-30 from left to right and from up downwards. Afterwards, these areas are examined and compared side by side with those of the control kneeprints to find out any typicality.
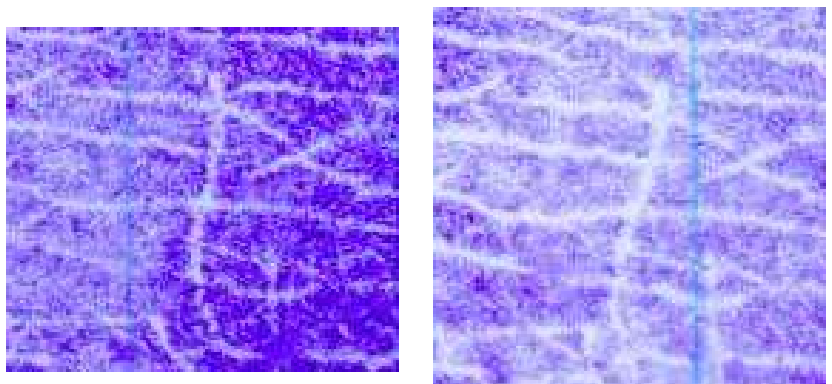

Figure 2: Rectangular areas of typical knee prints show that the shape of grooves-representing lines and shape of that single hair, crossing down the lines, are exactly the same in both prints. 
similarity in the configuration of the transverse and oblique groovesrepresenting lines (Figure 2-7).

Furthermore, they had shown some match in the width and shape of the spaces among the grooves-representing lines, which represent the flattened ridge areas, even in the position, size and shape of any existing hair/hairs or any detail else (Figure 8-13).

Matching areas were saved in pairs and re-examined again in a second session to make sure of the typicality and stability of such kind
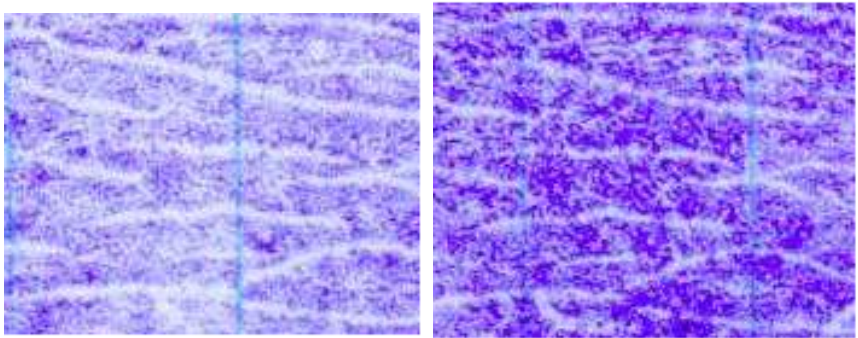

Figure 3: Typical rectangular areas of kneeprints show an exact configuration, which could not be found in any print else.
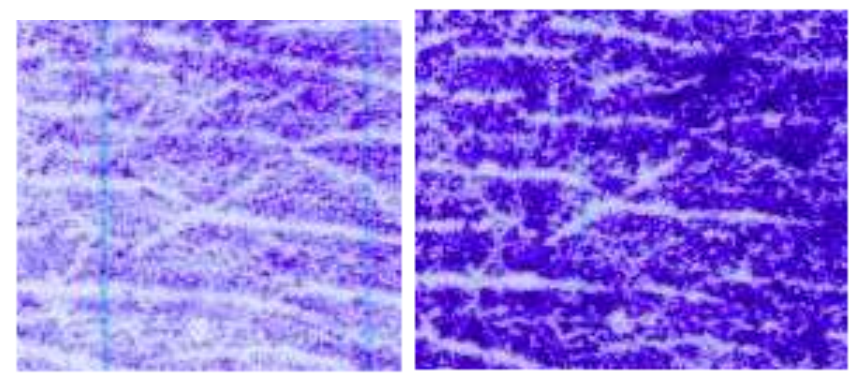

Figure 4: Typical match of two rectangular areas from the same kneeprint can be recognized at the end when their details have not been found in any print else.
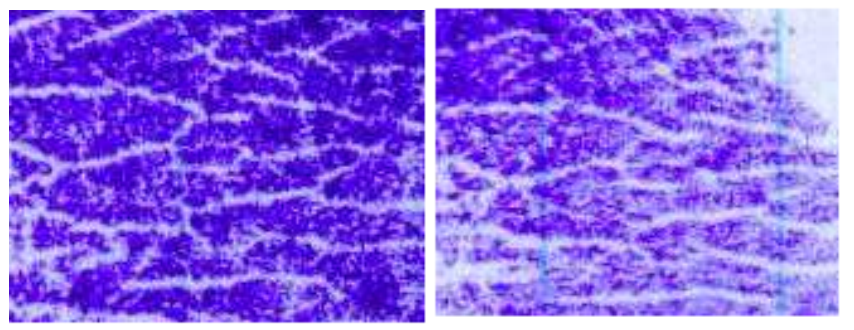

Figure 5: Despite the upper right corner of the lower rectangular area is mising under meticulous examination both typical areas are possible to be identified.
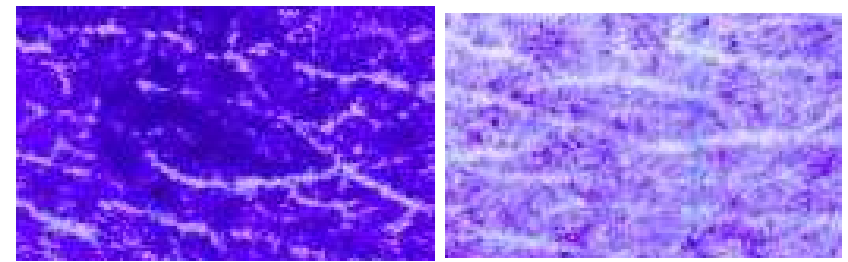

Figure 6: Despite the upper print area is not easily seen, comparable areas can be identified at the end. Computer software identification programs would be of great help in such circumstances.

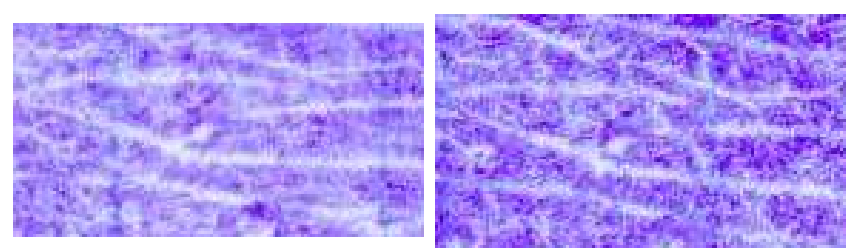

Figure 7: No one can argue about typicalıty of both areas, and it was so dittıcult to reach that degree of comparability among a large number of kneeprint areas.
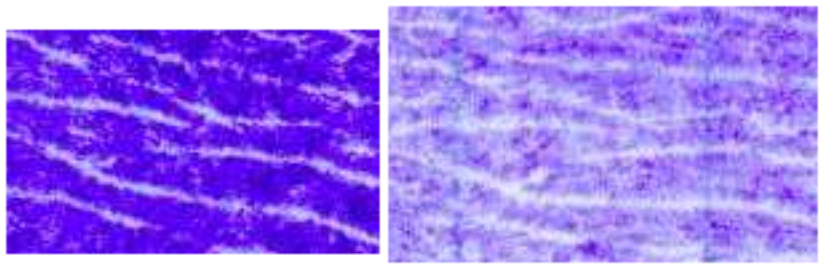

Figure 8: Despite the extradensity of colour of the upper kneeprint area, it was easy to compare and identify its typical one after a great deal of the meticulous examination.
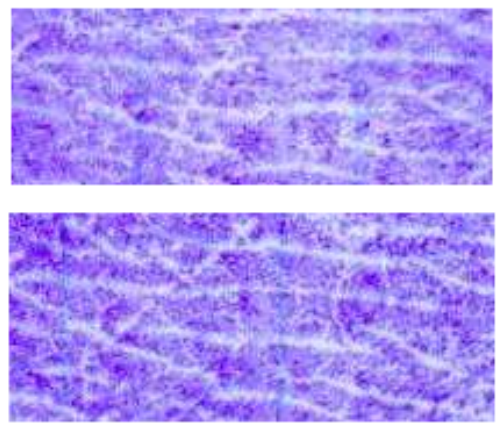

Figure 9: Despite the slight change in the shape and position of the single hair which is seen crossing down the print from above in the middle downwards to the left, it was not difficult to identify the two comparable kneeprint areas by their grooves-representing lines.
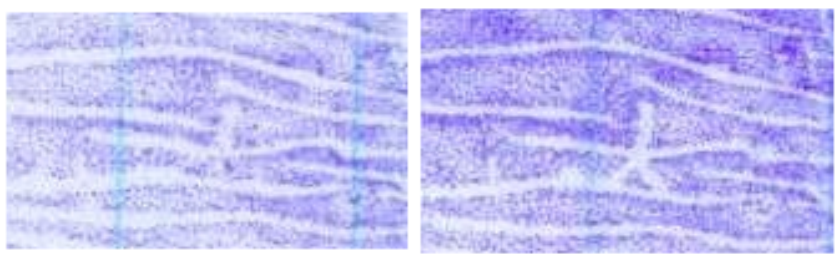

Figure 10: It is common to come across a print with a peculiar configuration in the grooves-representing lines (i.e. star shaped), so in such cases, it is easy to recognize the print and identify its comparable one.

of prints and any other clear areas of any two typical prints were further compared to confirm typicality.

\section{Discussion}

Despite absence of any previous work related to knee prints as a potential tool in identification, this does not rule out their potential role in personal identification especially in cases when other prints are missing. Knee print is actually a big-sized print relative to any print else and this makes it impossible to examine it as one mass this 

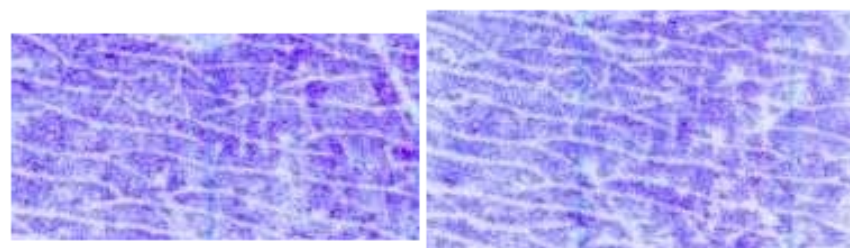

Figure 11: The most common problem with the kneeprint collection process is that any slight movement that might happen in the kneejoint during collection, or any slight increase or decrease of knee flexion during the collection, results in some changes in the print that necessitates extra interest to identify the typical prints, which are slightly different in shape.
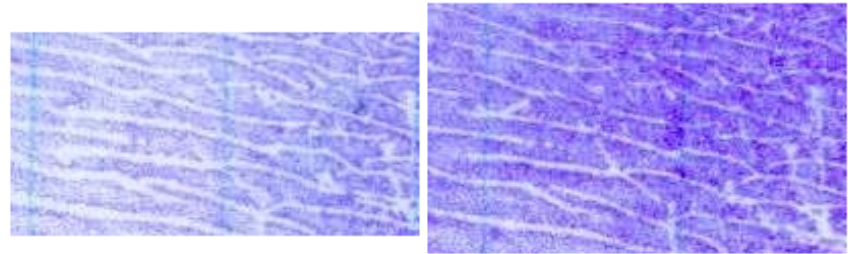

Figure 12: Some of the problems, which are commonly encountered during kneeprint identification are the disparity of color between both prints and or the thickening of grooves-representing lines of one print. They are due to the decrease or increase of either the dye concentration or the pressure exerted during print collection, respectively.

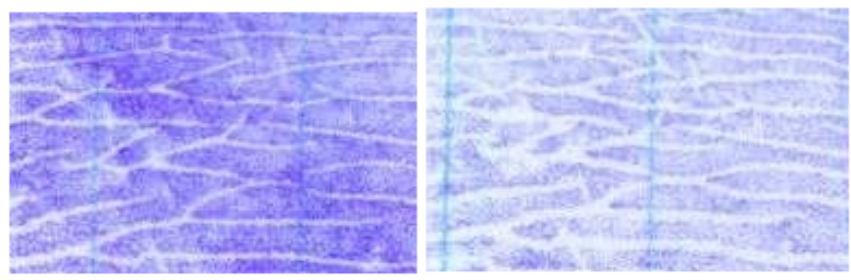

Figure 13: Despite disparity of color and slight change of the spaces between grooves-representing lines, by meticulous examination, comparable prints could be identified. The slight change in the space between grooves-representing lines results from any slight change of degree of knee flexion during collection of prints.

makes it different from fingerprints. Therefore, it was thought that its identification would better be achieved by dividing it into several rectangular areas and comparing such individual areas side by side after their magnification. The match in case of identification of any knee print can probably be considered when the typical match of one or more of its individual rectangular areas is attained. Knee prints, despite the problems confronted with their collection of having more or less mutilated areas, were suitable for examination, so they might be helpful in circumstances when it is necessary to resort to comparing such kind of prints. Under meticulous and detailed examination, each print looks, for some degree, distinguished from others in the configuration of its minute details at least at some areas.

The following points should be considered in case of comparing two knee prints: Shape of the lines representing grooves. Size and shape of the areas representing flattened ridges among the grooves lines. Any peculiarity in shape or any specific marks that might help in identification such as shape, size and direction of crossing hairs also should be considered.

Despite the difficulty of identifying a particular knee print rectangular area within more than thousand areas related to the other knee prints, it was possible by time and experience to get used to comparing different knee prints and identifying any including and excluding points. This work was considered as a pilot experiment just to check the possible variability of knee prints among different individuals and to study its potential role in personal identification. Therefore, testing was restricted to adult males only.

In the present paper, representing pilot study of knee print, the percentage of identified prints of the positive control group was 70\% (14 out of 20), however, this percentage would probably been improved by adopting the friction ridge enhancement measures and improving their collection methods.

\section{References}

1. http://hackney.tng.uk.com/biometric_systems/fingerprint.

2. Champod C (2004) Fingerprints and Other Ridge Skin Impressions. Boca Raton, FL: CRC Press.

3. Maltoni D, Maio D, Jain AK, Prabhakar S, (2003) Springer Verlag, Handbook of Fingerprint Recognition.

4. Cowger J (1983) Friction Ridge Skin Characteristics. Boca Raton: CRC Press

5. Burger M, Burger W (1998) "Ear biometrics." In Biometrics: Persona Identification in Networked Society ed. Jain A et al. Kluwer Academic Publishers.

6. Joshi PD, Shrestha MD (2002) Pneumococcal infection of a peosthetic knee joint. Infect Med 19: 517-521.

7. Pena E, Calvo B, Martinez MA, Doblare M (2005) A three-dimensional finite element analysis of the combined behavior of ligaments and menisci in the healthy human knee joint. J Biomech 29: pS0021-9290.

8. Pankanti S, Prabhakar S, Jain AK (2002) "On the individuality of fingerprints" IEEE Transactions on PAMI. 24: 1010-1025. 